\title{
Overweight and smoking promote recurrent lumbar disk herniation after discectomy
}

\author{
Alessandro Siccoli ${ }^{1}$. Victor E. Staartjes ${ }^{1,2,3}\left[\right.$ ] Anita M. Klukowska ${ }^{1,2} \cdot$ J. Paul Muizelaar ${ }^{4} \cdot$ Marc L. Schröder $^{1}$
}

Received: 14 May 2021 / Revised: 22 December 2021 / Accepted: 10 January 2022 / Published online: 24 January 2022

(c) The Author(s) 2022

\begin{abstract}
Purpose Recurrent lumbar disk herniation (rLDH) following lumbar microdiscectomy is common. While several risk factors for primary LDH have been described, risk factors for rLDH have only sparsely been investigated. We evaluate the effect of Body mass index (BMI) and smoking on the incidence and timing of rLDH.

Methods From a prospective registry, we identified all patients undergoing primary tubular microdiscectomy (tMD), with complete BMI and smoking data, and a minimum 12-month follow-up. We defined rLDH as reherniation at the same level and side requiring surgery. Overweight was defined as BMI $>25$, and obesity as BMI $>30$. Intergroup comparisons and age- and gender-adjusted multivariable regression were carried out. We conducted a survival analysis to assess the influence of BMI and smoking on time to reoperation.

Results Of 3012 patients, 166 (5.5\%) underwent re-microdiscectomy for rLDH. Smokers were reoperated more frequently $(6.4 \%$ vs. $4.0 \%, p=0.007)$. Similarly, rLDH was more frequent in obese $(7.5 \%)$ and overweight $(5.9 \%)$ than in normal-weight patients $(3.3 \%, p=0.017)$. Overweight smokers had the highest rLDH rate $(7.6 \%)$. This effect of smoking (Odds ratio: 1.63 , 96\% CI: 1.12-2.36, $p=0.010$ ) and BMI (Odds ratio: 1.09, 95\% CI: 1.02-1.17, $p=0.010$ ) persisted after controlling for age and gender. Survival analysis demonstrated that rLDH did not occur earlier in overweight patients and/or smokers.

Conclusions BMI and smoking may directly contribute to a higher risk of rLDH, but do not accelerate rLDH development. Smoking cessation and weight loss in overweight or obese patients ought to be recommended with discectomy to reduce the risk for rLDH.
\end{abstract}

Keywords Lumbar disk herniation · Discectomy $\cdot$ Recurrence $\cdot$ Lifestyle $\cdot$ Smoking $\cdot$ Overweight $\cdot$ Obesity

Alessandro Siccoli and Victor E. Staartjes have contributed equally to this work and share first authorship.

Alessandro Siccoli

alessandro.siccoli@gmail.com

$\triangle$ Victor E. Staartjes

victor.staartjes@gmail.com

1 Department of Neurosurgery, Bergman Clinics, Naarden Rijksweg 69, 1411 GE Naarden, Amsterdam, The Netherlands

2 Amsterdam UMC, Vrije Universiteit Amsterdam, Neurosurgery, Amsterdam Movement Sciences, Amsterdam, The Netherlands

3 Department of Neurosurgery, Clinical Neuroscience Center, University Hospital Zurich, University of Zurich, Zurich, Switzerland

4 Department of Neurological Surgery, Marshall University, Huntington, West Virginia, USA

\section{Introduction}

Sciatica due to lumbar disk herniation (LDH) represents one of the most frequent indications for lumbar spine surgery [1]. While patient satisfaction and rates of improvement in pain and functional impairment are usually very high after microdiscectomy, reoperations due to recurrent $\mathrm{LDH}(\mathrm{rLDH})$ are not uncommon, with incidences ranging between 2 and $11 \%$ [2-8]. Reoperations for rLDH can incur high direct and indirect health care-associated costs, as well as additional complications and morbidity $[6,7,9]$.

While risk factors for primary LDH have been relatively well-established, the literature is rather sparse on risk factors for rLDH. Still, several studies have investigated risk factors or potential etiologies for rLDH, however with often inconsistent results, low statistical power, or low effect sizes for certain potential risk factors [3, 7, 10-14]. Knowledge of robust risk factors for reherniation with a clinically relevant 
effect size would be clinically beneficial, especially if those risk factors were modifiable, such as "lifestyle" factors like smoking and body weight [15].

Multiple studies have assessed the effect of weight or Body mass index (BMI) on the incidence of rLDH. While some papers were able to detect an association between overweight and higher reoperation rates [14, 16], other studies identified no effect $[3,11-13,17,18]$. Some investigators have identified a positive association among smoking and reoperation rate $[3,11,13]$, while one paper found no difference [12]. However, the main limitation for almost all of these studies investigating the association of "lifestyle" factors smoking and body weight on rLDH demonstrated low sample sizes and consequently low statistical power, which is why additional studies with prospective and larger patient cohorts have been called for [3, 13]. In addition, no studies have assessed the influence of these risk factors on time to recurrence.

Identifying such risk factors and etiologies may not only help to better understand the nature of rLDH, but-in case of modifiable risk factors-may also lead to lower reoperation rates through optimal pre and postoperative patient management. Thus, any robust, modifiable "lifestyle" risk factor for rLDH would be clinically valuable. We evaluate the effect of BMI and smoking on the incidence and timing of rLDH after microdiscectomy.

\section{Materials and methods}

\section{Patient population}

We identified all patients who underwent primary tubular microdiscectomy (tMD) out of a prospective institutional registry of surgical interventions, as described previously [5]. All patients were operated on by a single neurosurgeon (M.L.S.)-focused solely on minimally invasive degenerative spine surgery-between 2010 and 2019 at a specialized, high-caseload, short-stay spine center [19]. Indications for surgery-whether for index or redo cases-have been reported previously $[5,20]$. This report was compiled according to the "Strengthening the reporting of observational studies in epidemiology" (STROBE) guidelines [21].

Preoperative inclusion criteria were single-level LDH confirmed using magnetic resonance imaging and failed conservative management for $\geq 8$ weeks [22]. Reoperations were performed in patients with a recurrence of disabling pain and/or neurological symptoms, who failed conservative therapy and in whom a MRI showed rLDH. All consecutive cases of first-time tMD for LDH with a follow-up threshold of $\geq 12$ months regarding recurrence (surgery at least 12 months before this analysis), as well as complete data on either one or both of (1) preoperative BMI and/or (2) smoking status were included in our analysis to maximize statistical power with the available prospective registry data. Patients who had received prior surgery at the index level (e.g., those with a previous history of discectomy who were referred to us from another center) were excluded.

\section{Ethical considerations}

This institutional registry was approved by the local institutional review board (Medical Research Ethics Committees United, Registration Number: W17.067). Additionally, all individual patients included in the study provided written informed consent, and the study was performed according to the Declaration of Helsinki and its amendments.

\section{Outcomes and data collection}

We defined rLDH as a return of preoperative pain symptoms after a pain-free period after primary discectomy, combined with ipsilateral nerve root compression at the same level, visible on magnetic resonance imaging that necessitated surgical revision [4, 5, 23]. Additionally, the period between primary microdiscectomy and reoperation was measured in days for each patient with rLDH. Any reoperations-including reoperation for $\mathrm{LDH}$-were systematically tracked and entered into the registry.

Weight and height were measured preoperatively and entered into the prospective patient registry. According to the WHO classification, overweight was defined as a BMI of $25.0 \mathrm{~kg} / \mathrm{m}^{2}$ or greater, and obesity was defined as a BMI of $30.0 \mathrm{~kg} / \mathrm{m}^{2}$ or greater [24]. Smoking status was captured preoperatively, although we did not record how long before surgery patients had given up smoking. "Non-smokers" included never-smokers and individuals who had ceased smoking preoperatively.

\section{Statistical analysis}

Continuous data are given as mean \pm standard deviation or median (Interquartile range, IQR), and categorical data as numbers (percentages).

Pearson's $\chi^{2}$ test was conducted to assess the association of smoking status and body weight classes with rLDH. Median time to recurrence was compared using the exact version of Wilcoxon's rank-sum test, based on the "shift" algorithm described by Streitberg and Röhmel [25]. In addition, we performed an age- and sex-adjusted multivariable logistic regression analysis to determine the influence of smoking status and BMI on rLDH while controlling for these potential confounders.

The effect of smoking and overweight on recurrence was also determined using an age- and sex-adjusted Cox proportional hazards model. The log-rank test was used to test 
for significance in the survival analyses. Patients without reoperation for rLDH were right-censored at the date of last follow-up, and Kaplan-Meier curves were constructed. For all analyses, a $p \leq 0.05$ was considered as statistically significant on two-tailed tests. All analyses were carried out using $\mathrm{R}$ version 3.6.1 (The R Foundation for Statistical Computing, Vienna, Austria) [26].

\section{Results}

\section{Patient cohort}

A total of 3012 patients included in the prospective registry underwent primary tMD for LDH during the study period (Fig. 1). All 3012 patients had a minimum 12-month followup with data on reoperations, since all patients were followed clinically for at least a year and since we only include patients who were operated at least one year before the initiation of this analysis according to our inclusion criteria. Overall, 166 of 3012 patients (5.5\%) had to undergo reoperation for rLDH, with $798(31 \%)$ active smokers and a mean BMI of $25.5 \pm 3.5 \mathrm{~kg} / \mathrm{m}^{2}$. Detailed baseline patient characteristics are provided in Table 1. From this database we analyzed 2584 patients with complete smoking data, 1481 patient with complete BMI data, and 1335 with combined smoking and BMI data. Patients had to undergo reoperation at an average of $476 \pm 525$ days. Baseline characteristics were compared among the subgroups in Supplementary Tables 1 and 2.

\section{Association of BMI with recurrence}

Rates of rLDH stratified by BMI and by smoking status are provided in Table 2 . The incidence of reoperation for rLDH was higher in overweight (5.9\%) and obese (7.5\%) patients compared to normal-weight patients $(3.3 \%, p=0.017$, Fig. 2). After correction for age and gender, the positive relationship among BMI and rLDH persisted (Odds Ratio $(\mathrm{OR})=1.09,95 \%$ confidence interval $(\mathrm{CI})=1.02-1.17$, $p=0.010$, Table 3). A survival analysis adjusted for age and gender also demonstrated this effect (Fig. 3) with a hazard ratio of 1.899 for overweight versus normal-weight patients $(p=0.011)$. Overweight patients experienced rLDH after a similar number of days $(N=48$, Median $=343$ days, $\mathrm{IQR}=146$ to 661 days) as their normal-weight counterparts $(N=24$, Median $=245$ days, $\mathrm{IQR}=142$ to 637 days, $p=0.555)$.

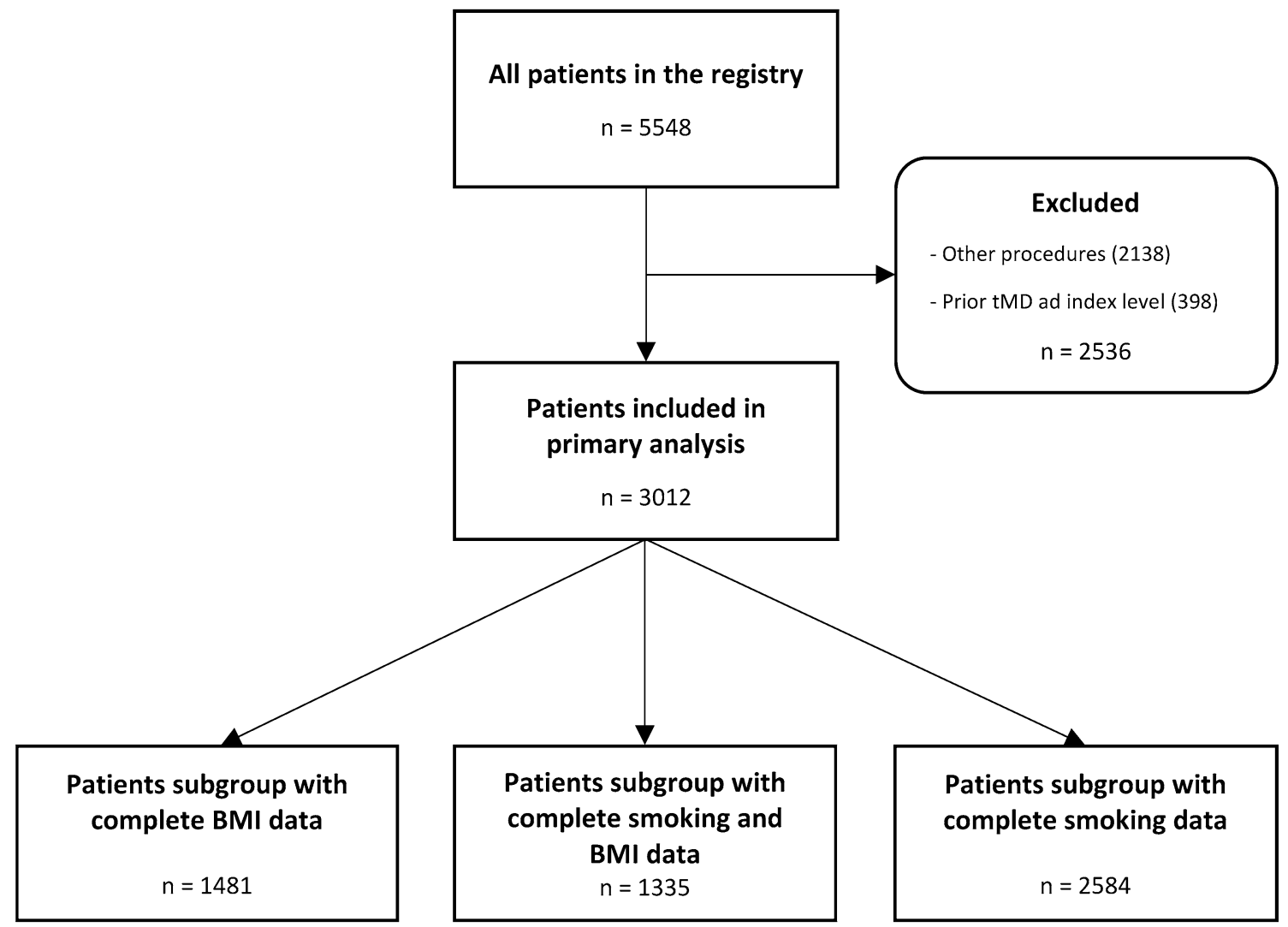

Fig. 1 Flowchart demonstrating the flow of patients throughout this analysis 
Table 1 Baseline patient characteristics

\begin{tabular}{ll}
\hline Variable & Value \\
& $N=3012$ \\
\hline Age [years] & $45 \pm 13$ \\
Active smoker & $798(31 \%)$ \\
Male gender & $1608(53 \%)$ \\
BMI [kg/m $\left.{ }^{2}\right]$ & $25.5 \pm 3.5$ \\
ASA score & \\
Class I & $1762(64 \%)$ \\
Class II & $931(36 \%)$ \\
Class III & $7(0.2 \%)$ \\
Index level & \\
L1-L2 & $4(0.1 \%)$ \\
L2-L3 & $44(1.4 \%)$ \\
L3-L4 & $187(6.2 \%)$ \\
L4-L5 & $1344(45 \%)$ \\
L5-S1 & $1433(48 \%)$ \\
Side & \\
Right-sided & $1493(49.6 \%)$ \\
Left-sided & $1376(45.7 \%)$ \\
Midline & $102(3.4 \%)$ \\
Bilateral & $41(1.4 \%)$ \\
Reoperation for rLDH & $166(5.5 \%)$ \\
Time to reoperation [days] & $476 \pm 525$ \\
\hline
\end{tabular}

$B M I$, Body mass index; $A S A$, American society of anesthesiologists; $r L D H$, Recurrent lumbar disk herniation

Table 2 Confrontation of rLDH rates for active smokers compared to non-smokers, as well as for $\mathrm{BMI}<25$ (normal-weight) compared to 25-30 (overweight) and $>30 \mathrm{~kg} / \mathrm{m}^{2}$ (obesity), and for the combination of both potential risk factors

\begin{tabular}{llll}
\hline Variable & Number & rLDH & $p$ value \\
\hline Smoking & $N=2584$ & \multicolumn{2}{l}{$0.007^{*}$} \\
Non-smoker & $1786(69 \%)$ & $71(4.0 \%)$ & \\
Active Smoker & $798(31 \%)$ & $51(6.4 \%)$ & \\
BMI & $N=1481$ & & $0.017^{*}$ \\
$<25 \mathrm{~kg} / \mathrm{m}^{2}$ & $703(47 \%)$ & $23(3.3 \%)$ & \\
$25-29.9 \mathrm{~kg} / \mathrm{m}^{2}$ & $592(40 \%)$ & $35(5.9 \%)$ & \\
$\geq 30 \mathrm{~kg} / \mathrm{m}^{2}$ & $186(13 \%)$ & $14(7.5 \%)$ & \\
Smoking and BMI & $N=1335$ & & $0.038^{*}$ \\
Non-smoker and BMI $<25$ & $444(33 \%)$ & $14(3.2 \%)$ & \\
Active smoker or BMI $\geq 25 \mathrm{~kg} /$ & $693(52 \%)$ & $29(4.2 \%)$ & \\
$\quad \mathrm{m}^{2}$ & & & \\
Active smoker and BMI $\geq 25 \mathrm{~kg} /$ & $198(15 \%)$ & $15(7.6 \%)$ & \\
$\mathrm{m}^{2}$ & & & \\
\hline
\end{tabular}

${ }^{*} p \leq 0.05$

\section{Association of smoking with recurrence}

Smokers had a significantly higher incidence of rLDH
(6.4\%) compared to non-smokers $(4.0 \%, p=0.007)$. In the multivariable logistic regression analysis corrected for age and gender, smoking independently predicted $\mathrm{rLDH}$ $(\mathrm{OR}=1.63,95 \% \mathrm{CI}=1.12-2.36, p=0.010$, Table 3$)$. The age- and gender-adjusted survival analysis corroborated this finding (Hazard ratio $=1.591, p=0.012$, Fig. 3), and smokers experienced rLDH after a similar duration $(N=51$, Median $=350$ days, $\mathrm{IQR}=135$ to 781 days) as non-smokers $(N=71$, Median $=237$ days, IQR $=111$ to 521 days, $p=0.129)$.

\section{Association of combined smoking and overweight with recurrence}

To assess any potential accumulative effect of combined active smoking and overweight, we carried out an additional analysis among the 1335 (44\%) patients with complete data on both potential risk factors (Fig. 4). Active smokers with a BMI $\geq 25.0 \mathrm{~kg} / \mathrm{m}^{2}$ had the highest rate of rLDH (7.6\%, Table 4). Patients with both potential risk factors also demonstrated a hazard ratio of $1.91(95 \% \mathrm{CI}=1.06-3.45$, $p=0.032$ ) for development of $\mathrm{rLDH}$ requiring reoperation in the adjusted survival analysis. These patients also experienced rLDH after a similar number of days $(N=15$, Median $=456$ days, $\mathrm{IQR}=272$ to 959 days) than others $(N=43$, Median $=245$ days, IQR $=138$ to 613 days, $p=0.078)$.

\section{Discussion}

Using a large patient sample from a prospective institutional registry, the association of the two "lifestyle" factors body weight-captured as BMI-and active smoking with the incidence and timing of symptomatic rLDH requiring reoperation was evaluated. Out of all patients, around five percent necessitated reoperation for recurrent herniation. Both active smoking and overweight led to consistent and clinically relevant increases in the incidence of rLDH. Multivariable logistic and survival modeling corroborated these findings. A combination of the two risk factors led to the highest odds of developing rLDH requiring reoperation. However, active smoking and overweight do not appear to influence the timing of rLDH requiring surgical treatment.

Symptomatic recurrent disk herniation is relatively common after primary microdiscectomy, often requires patients to undergo redo surgery which puts them at excess risk for other surgical and medical complications, and also incurs high direct and indirect health care costs [9]. The reoperation rate of 5.5\% after primary tubular microdiscectomy conforms with values ranging between 3 and $13 \%$ in the contemporary literature [2-8]. Preoperative risk assessment can be beneficial for improved patient counseling and 

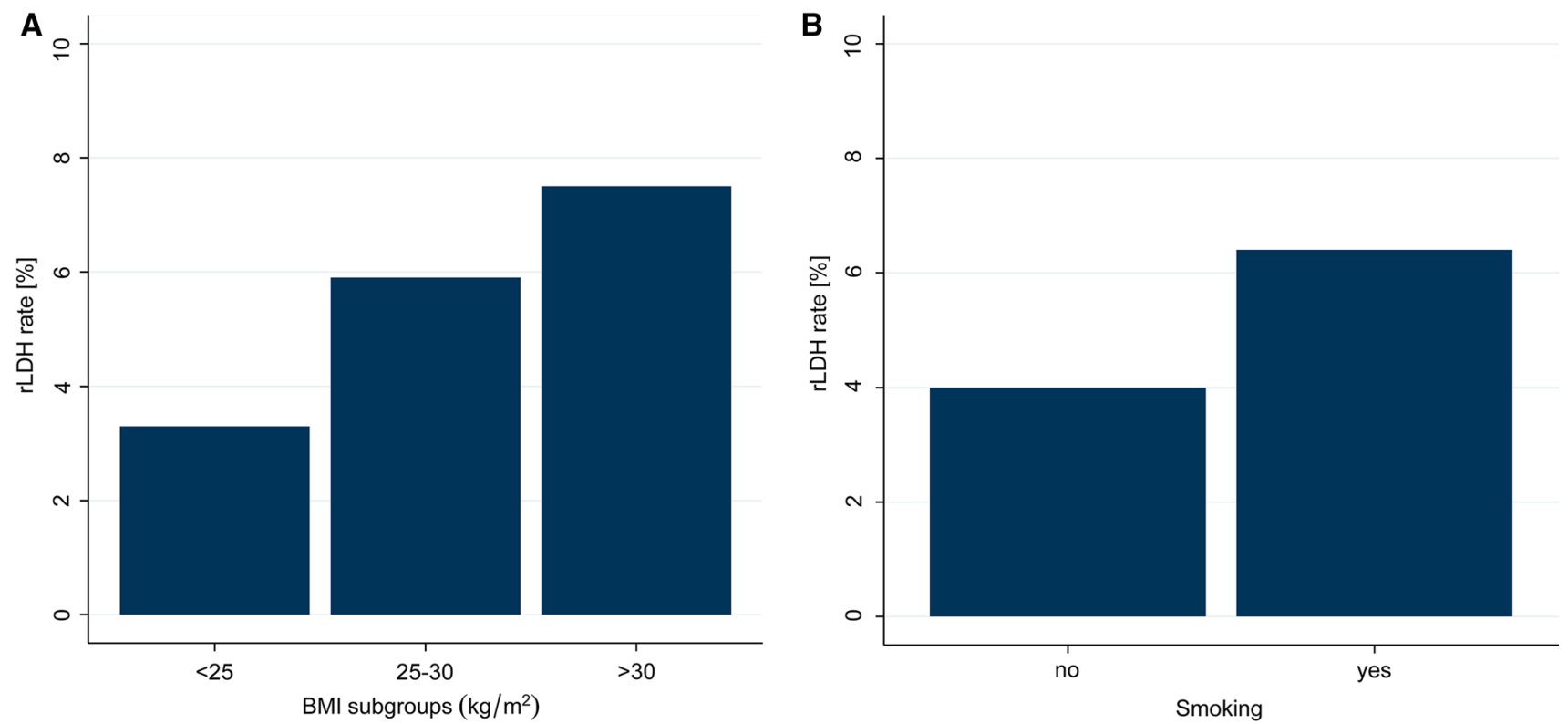

Fig. 2 Rates of recurrent lumbar disk herniation (rLDH) among the studied subgroups. Panel A demonstrates rLDH rates for normal-weight, overweight, and obese patients. Panel B shows rLDH rates for non-smokers and smokers

Table 3 Logistic regression values with $\mathrm{rLDH}$ as dependent variable and BMI or smoking, respectively, as well as age and gender as independent variables. Each one-step increase in BMI led to a 1.09-fold increased odds of rLDH, and active smokers had 1.63-fold higher odds of rLDH compared to non-smokers

\begin{tabular}{llll}
\hline Variable & $\begin{array}{l}\text { Odds Ratio } \\
(N=1481)\end{array}$ & $95 \%$ CI & $P$ value \\
\hline BMI subgroup & & & \\
$\quad$ BMI & 1.09 & $1.02-1.17$ & $0.010^{*}$ \\
Male gender & 1.16 & $0.72-1.88$ & 0.553 \\
Age & 1.00 & $0.98-1.01$ & 0.603 \\
Smoking subgroup & & & \\
Active smoker & 1.63 & $1.12-2.36$ & $0.010^{*}$ \\
Male gender & 1.19 & $0.82-1.73$ & 0.362 \\
Age & 1.00 & $0.98-1.01$ & 0.899 \\
\hline
\end{tabular}

${ }^{*} p \leq 0.05$

$r L D H$, Recurrent lumbar disk herniation; $B M I$, body mass index; $C I$, Confidence interval

individualized risk-benefit discussion [15, 27], although patient-specific risk factors for reherniation would only very rarely dissuade from surgery once a clear indication for surgery is given. This is why-apart from potentially contributing to the understanding of the etiopathology of recurrence-risk factors are the most clinically relevant if they are modifiable [15].

Several studies have investigated the effect of BMI or smoking on rLDH, with conflicting results. Most of these studies have included smaller cohorts of less than 300 patients and correspondingly even fewer patients with rLDH, with consequently less powerful effect estimates $[3,11,14,18]$. A meta-analysis conducted by Huang et al. [13] demonstrated an increased risk for rLDH in smokers $(\mathrm{OR}=1.99,95 \% \mathrm{CI}=1.53-2.58)$, and no effect of overweight $(\mathrm{OR}=1.11,95 \% \mathrm{CI}=0.57-2.16)$ based on only two studies, with relatively low heterogeneity. Our findings demonstrate a recurrence-promoting effect of both overweight and active smoking with higher statistical power and prospective data, which corresponds to the results of the majority of studies [3, 11, 13, 18]. Furthermore, the accumulative effect of the two risk factors observed in our data further corroborates their potential to causatively promote $\mathrm{rLDH}$ ("biological gradient") [28].

Still, the nature of the effect of smoking on rLDH pathophysiology remains poorly understood. In an experimental biological study with mice, smoking has been shown to lead to misalignment and lesions of the annulus fibrosus [29]. In addition, the vasoconstrictor effect of nicotine may also inhibit the synthesis of proteoglycans, consequently leading to a more vulnerable disk [30,31]. Lastly, chronic cough as a consequence of smoking was also shown to increase intradiscal pressure and therefore potentially lead to recurrent disk herniations, especially when also considering the effects of smoking on wound healing in other domains [32-34].

The effect of overweight on rLDH in the literature remains more unclear $[3,4,11,13,14,16-18]$. Overweight and especially obesity have been well-described to be an independent risk factor for degenerative disease, including disk herniation as well as degenerative disk disease 


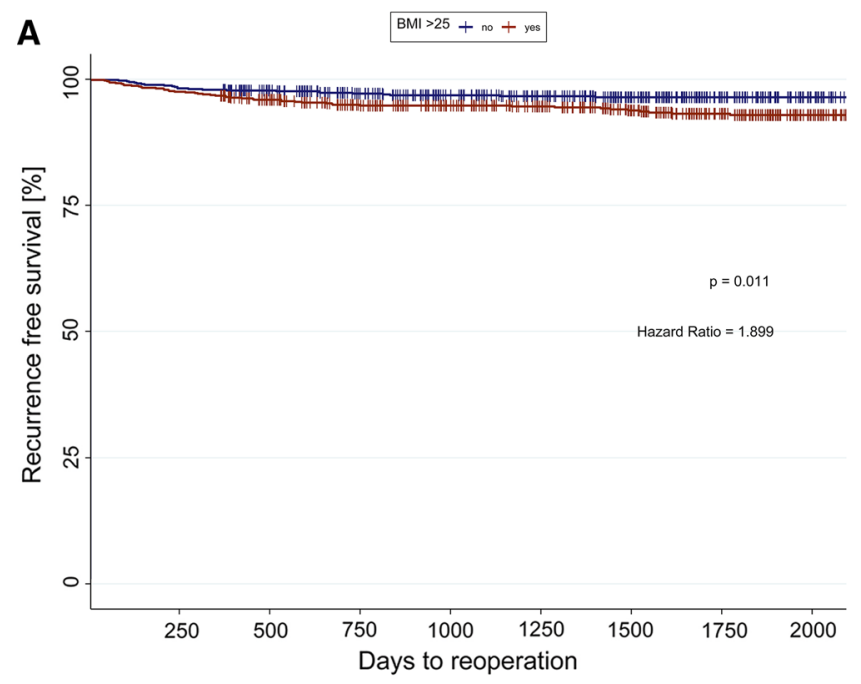

Fig. 3 Kaplan-Meier estimates for recurrence-free survival are shown for normal-weight versus overweight patients (Panel A), as well as for non-smokers versus smokers (Panel B). Data are rightcensored (vertical lines) according to the duration of clinical follow-

and spondylolysis [35-39]. These effects may be partially explained by the elevated mechanical load on the spine that comes along with obesity, especially with abdominal obesity [40]. Obese patients also tend to undergo changes in posture and flexibility of the thoracolumbar spine, resulting in postures that increase the mechanical load on the spine [41]. On the other hand, adipose tissue produces and releases adipokines such as leptin and adiponectin, which in turn induce inflammatory and degrading processes leading to disk degeneration $[42,43]$. A study investigating glycosaminoglycan (GAG) concentration in the nucleus pulposus found decreased GAG levels in obese patients [44], which may correspond to morphological finding in degenerated intervertebral disk [44, 45]. Overall, obese patients also empirically appear to be less likely to experience clinically meaningful symptomatic improvement after discectomy [46, 47].

While several studies have previously investigated factors associated with $\mathrm{rLDH}$, none have analyzed the timing of recurrence. In our study, active smokers and overweight patients did not significantly differ in the speed of their progression to rLDH compared to their non-smoking and normal-weight counterparts.

In combination with the literature, our findings suggest that both overweight-especially obesity-and active smoking independently promote symptomatic rLDH requiring reoperation. The effect sizes, which were reproduced through multiple analytical methods, further corroborate the potential clinical relevance of our findings: Active smoking led to approximately 1.6-fold increased absolute risk of recurrence, while overweight and obesity led to approximately

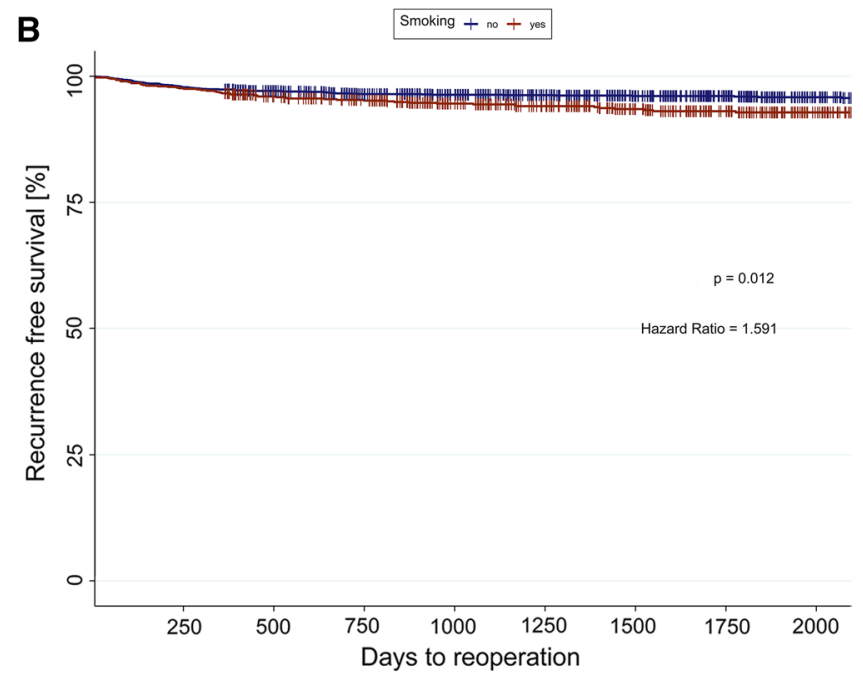

up. Additionally, the statistical results of the age- and sex-adjusted survival analysis of time to recurrent lumbar disk herniation (rLDH) are presented

1.8-fold and 2.3-fold increased absolute risks of recurrence, respectively. Reoperations for recurrence are inconvenient and costly, and any preventative measures to prevent recurrence requiring redo surgery ought to be considered. In recent years, even implantable devices have been trialed to reduce the risk of symptomatic reherniation requiring surgery, with mixed results [23]. Lifestyle risk factors that are modifiable and that have a robust and clinically relevant effect could be very valuable in reducing the incidence of rLDH. Further studies evaluating structured interventions for weight loss and smoking cessation before discectomy should be carried out to assess if these measures can be truly clinically impactful.

\section{Strengths and limitations}

The main strengths of this analysis are that its source data originate from a prospective institutional registry, and that the statistical power is very high due to available sample size with complete data. Furthermore, reoperations were systematically tracked. Moreover, we observed large and consistent effect sizes for the associations of BMI and smoking with rLDH, among multiple statistical methods, and there is at least some biological plausibility to support the main findings [28].

The main limitation however remains the retrospective nature of this specific analysis, as selection bias cannot be ruled out, although all data was collected prospectively and all patients with sufficient data were included. Even though we examined a large prospective cohort, the sample 


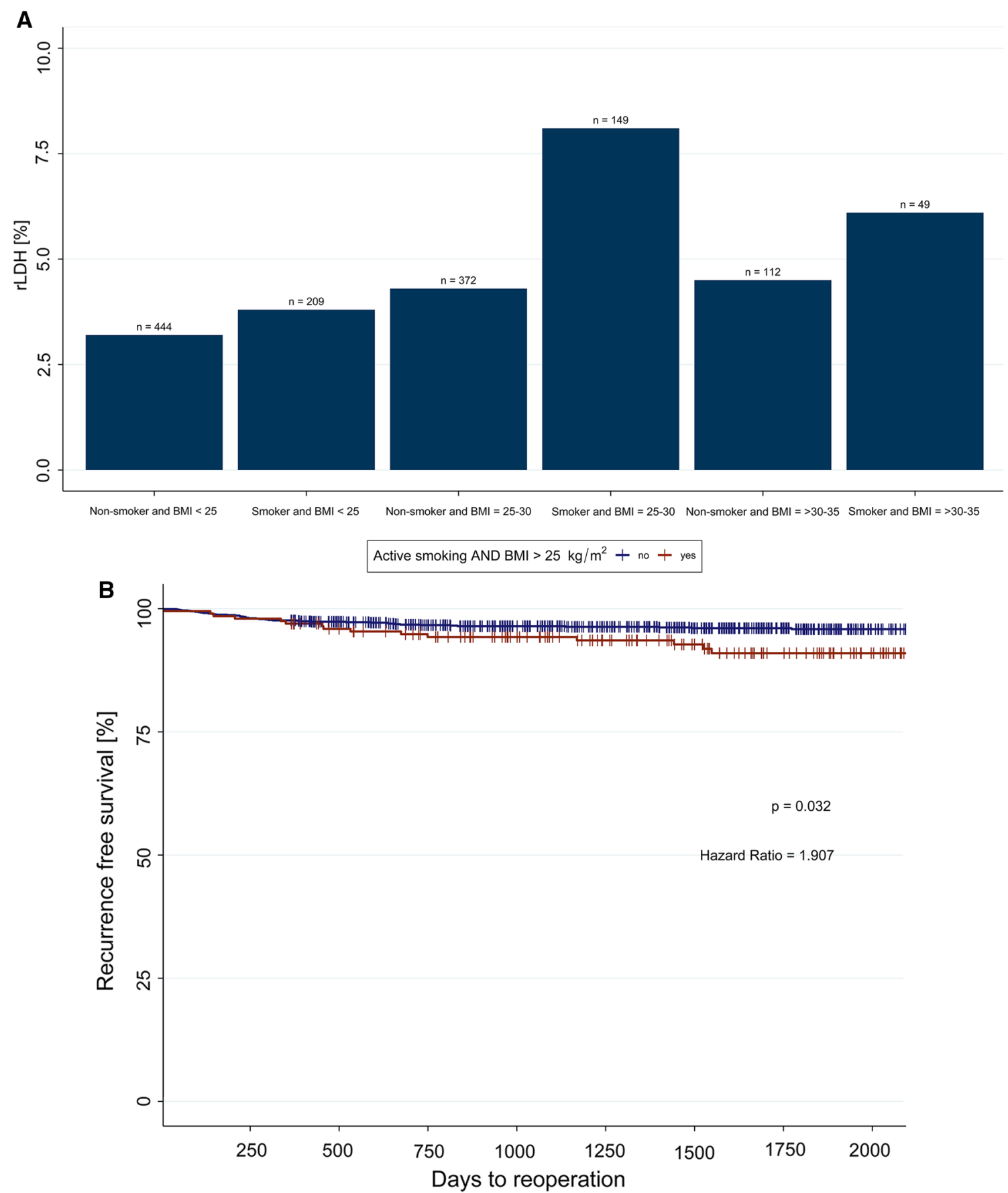

Fig. 4 Panel A demonstrates rates of recurrent lumbar disk herniation ( $\mathrm{rLDH})$ for patients with different combination of the two studied risk factors. Kaplan-Meier estimates for recurrence-free survival are shown for patients who are both active smokers and overweight ver-

size and consequently power for analysis of time to recurrence was comparatively low. All data stem from a single center, therefore center bias cannot be ruled out. On the other hand, single-surgeon or single-institution study designs eliminate variability in indications or techniques as factors contributing to recurrence. For example, out of sus others (Panel B). Data are right-censored (vertical lines) according to the duration of clinical follow-up. Additionally, statistical results of the age- and sex-adjusted survival analysis of time to rLDH are provided

surgeon preference, our cohort includes only tubular discectomy cases, which however have been demonstrated to lead to equal outcomes compared to conventional microdiscectomy [48]. Because of local insurance regulation restrictions, patients aged $>80$, with an American Society of Anesthesiologists (ASA) score $>2$ or with a body 
Table 4 Logistic regression values with rLDH as dependent variable and the combination of active smoking with overweight, age and gender as independent variables. Active smokers who were also overweight or obese had 1.99-fold higher odds of rLDH compared to others

\begin{tabular}{llll}
\hline Variable & $\begin{array}{l}\text { Odds Ratio } \\
(\mathrm{N}=1335)\end{array}$ & $95 \% \mathrm{CI}$ & P value \\
\hline $\begin{array}{llll}\text { Active smoker and } \\
\mathrm{BMI} \geq 25 \mathrm{~kg} / \mathrm{m}^{2}\end{array}$ & 1.99 & $1.05-3.59$ & $0.027^{*}$ \\
Male gender & 1.39 & $0.81-2.43$ & 0.235 \\
Age & 0.99 & $0.97-1.01$ & 0.481 \\
\hline
\end{tabular}

${ }^{*} p \leq 0.05$

$r L D H$, Recurrent lumbar disk herniation; BMI, Body mass index; $C I$, Confidence interval

mass index $(\mathrm{BMI})>35$ were not allowed to be operated on in such a setting. Because of these local insurance regulations, patients with very severe comorbidities or very elderly patients were not included in this study, thus our findings concerning obesity can not be extrapolated to patients suffering from class II or III obesity. Because Gadolinium was not routinely used in patients with a clinical suspicion of LDH recurrence, it may be possible that some clinically judged rLDHs had a scar tissue component. To assess the incidence of the modifiable risk factors in a "non-regulated" environment we looked at 100 consecutive spine surgeries in a random time period by one of the authors (JPM), and found a higher incidence of smoking (36\%) and a much higher BMI (mean $33.7 \pm 7.7$, range 21-54, median 32). We also did not study the effects of smoking cessation or smoking quantity on LDH recurrence, nor those of diabetes mellitus or other potentially relevant variables such as disk height or type of job. Lastly, as with primary LDH, many recurrent herniations are asymptomatic [10], but we did not track patients with recurrent pain in whom redo surgery was not deemed indicated. Consequently, our findings may not necessarily extrapolate to those asymptomatic cases, but can instead only be applied to patients with symptomatic rLDH.

\section{Conclusions}

In an analysis of a large prospective institutional registry, we found that both overweight and active smoking likely contribute to a higher incidence of symptomatic rLDH requiring reoperation, but not necessarily to a more rapid development of reherniation. Modification of risk factors-through lifestyle interventions such as smoking cessation and weight loss in overweight or obese patients-may help reduce the incidence of reoperation for $\mathrm{LLDH}$.
Supplementary Information The online version contains supplementary material available at https://doi.org/10.1007/s00586-022-07116-y.

Funding Open access funding provided by University of Zurich. This research did not receive any specific grant from funding agencies in the public, commercial, or not-for-profit sectors.

\section{Declarations}

Conflict of interest The authors declare that the article and its content were composed in the absence of any commercial or financial relationships that could be construed as a potential conflict of interest.

Ethics approval W17.067, approved on 17.05 .2019 by Medical Research Ethics Committees United.

Informed consent All patients provided written informed consent.

Open Access This article is licensed under a Creative Commons Attribution 4.0 International License, which permits use, sharing, adaptation, distribution and reproduction in any medium or format, as long as you give appropriate credit to the original author(s) and the source, provide a link to the Creative Commons licence, and indicate if changes were made. The images or other third party material in this article are included in the article's Creative Commons licence, unless indicated otherwise in a credit line to the material. If material is not included in the article's Creative Commons licence and your intended use is not permitted by statutory regulation or exceeds the permitted use, you will need to obtain permission directly from the copyright holder. To view a copy of this licence, visit http://creativecommons.org/licenses/by/4.0/.

\section{References}

1. Ropper AH, Zafonte RD (2015) Sciatica. N Engl J Med 372:1240 1248. https://doi.org/10.1056/NEJMra1410151

2. Hlubek RJ, Mundis GM (2017) Treatment for Recurrent Lumbar Disc Herniation. Curr Rev Musculoskelet Med 10:517-520. https://doi.org/10.1007/s12178-017-9450-3

3. Shimia M, Babaei-Ghazani A, Sadat BE et al (2013) Risk factors of recurrent lumbar disk herniation. Asian J Neurosurg 8:93-96. https://doi.org/10.4103/1793-5482.116384

4. Moliterno JA, Knopman J, Parikh K et al (2010) Results and risk factors for recurrence following single-level tubular lumbar microdiscectomy: Clinical article. J Neurosurg Spine 12:680-686. https://doi.org/10.3171/2009.12.SPINE08843

5. Staartjes VE, de Wispelaere MP, Miedema J, Schröder ML (2017) Recurrent Lumbar Disc Herniation After Tubular Microdiscectomy: Analysis of Learning Curve Progression. World Neurosurg 107:28-34. https://doi.org/10.1016/j.wneu.2017.07.121

6. Shepard N, Cho W (2019) Recurrent Lumbar Disc Herniation: A Review. Global Spine Journal 9:202-209. https://doi.org/10.1177/ 2192568217745063

7. Lebow RL, Adogwa O, Parker SL et al (2011) Asymptomatic same-site recurrent disc herniation after lumbar discectomy: results of a prospective longitudinal study with 2-year serial imaging. Spine 36:2147-2151. https://doi.org/10.1097/BRS.0b013 e3182054595

8. Takahashi H (2014) Recurrent Lumbar Disc Herniation after Microendoscopic Discectomy. J Spine Neurosurg. https://doi.org/ 10.4172/2325-9701.1000141 
9. Selva-Sevilla C, Ferrara P, Gerónimo-Pardo M (2019) Cost-utility Analysis for Recurrent Lumbar Disc Herniation: Conservative Treatment Versus Discectomy Versus Discectomy With Fusion. Clin Spine Surg 32:E228-E234. https://doi.org/10.1097/BSD. 0000000000000797

10. McGirt MJ, Eustacchio S, Varga P et al (2009) A prospective cohort study of close interval computed tomography and magnetic resonance imaging after primary lumbar discectomy: factors associated with recurrent disc herniation and disc height loss. Spine 34:2044-2051. https://doi.org/10.1097/BRS.0b013e3181b34a9a

11. Miwa S, Yokogawa A, Kobayashi T et al (2015) Risk factors of recurrent lumbar disk herniation: a single center study and review of the literature. J Spinal Disord Tech 28:E265-269. https://doi. org/10.1097/BSD.0b013e31828215b3

12. Abdu RW, Abdu WA, Pearson AM et al (2017) Reoperation for Recurrent Intervertebral Disc Herniation in the Spine Patient Outcomes Research Trial: Analysis of Rate, Risk Factors, and Outcome. Spine 42:1106-1114. https://doi.org/10.1097/BRS.00000 00000002088

13. Huang W, Han Z, Liu J et al (2016) Risk Factors for Recurrent Lumbar Disc Herniation. Medicine (Baltimore). https://doi.org/ 10.1097/MD.0000000000002378

14. Meredith DS, Huang RC, Nguyen J, Lyman S (2010) Obesity increases the risk of recurrent herniated nucleus pulposus after lumbar microdiscectomy. The Spine Journal 10:575-580. https:// doi.org/10.1016/j.spinee.2010.02.021

15. Steinmetz MP, Mroz T (2018) Value of Adding Predictive Clinical Decision Tools to Spine Surgery. JAMA Surg. https://doi.org/10. 1001/jamasurg.2018.0078

16. Kim J, Lee S-H, Ahn Y et al (2007) Recurrence after Successful Percutaneous Endoscopic Lumbar Discectomy. Minimally invasive neurosurgery : MIN 50:82-85. https://doi.org/10. 1055/s-2007-982504

17. Kim K-T, Lee D-H, Cho D-C et al (2015) Preoperative Risk Factors for Recurrent Lumbar Disk Herniation in L5-S1. J Spinal Disord Tech 28:E571-577. https://doi.org/10.1097/BSD.00000 00000000041

18. Kim K-T, Park S-W, Kim Y-B (2009) Disc height and segmental motion as risk factors for recurrent lumbar disc herniation. Spine 34:2674-2678. https://doi.org/10.1097/BRS.0b013e3181b4aaac

19. Staartjes VE, de Wispelaere MP, Schröder ML (2019) Improving recovery after elective degenerative spine surgery: 5-year experience with an enhanced recovery after surgery (ERAS) protocol. Neurosurg Focus 46:E7. https://doi.org/10.3171/2019.1.FOCUS 18646

20. Siccoli A, Staartjes VE, de Wispelaere MP, Schröder ML (2019) Association of time to surgery with leg pain after lumbar discectomy: is delayed surgery detrimental? J Neurosurg. https://doi.org/ 10.3171/2019.8.SPINE19613

21. Von Elm E, Altman DG, Egger M et al (2007) Strengthening the Reporting of Observational Studies in Epidemiology (STROBE) statement: guidelines for reporting observational studies. BMJ 335:806-808. https://doi.org/10.1136/bmj.39335.541782.AD

22. Peul WC, van Houwelingen HC, van den Hout WB et al (2007) Surgery versus Prolonged Conservative Treatment for Sciatica. N Engl J Med 356:2245-2256. https://doi.org/10.1056/NEJMoa0640 39

23. Thomé C, Klassen PD, Bouma GJ et al (2018) Annular closure in lumbar microdiscectomy for prevention of reherniation: a randomized clinical trial. Spine J 18:2278-2287. https://doi.org/10. 1016/j.spinee.2018.05.003

24. Aronne LJ (2002) Classification of Obesity and Assessment of Obesity-Related Health Risks. Obes Res 10:105S-115S. https:// doi.org/10.1038/oby.2002.203
25. Streitberg B, Röhmel J (1986) Exact distributions for permutation and rank tests: an introduction to some recently published algorithms. Stat Software Newsletter 12:10-17

26. R Core Team (2020) R: A Language and Environment for Statistical Computing. R Foundation for Statistical Computing, Vienna, Austria

27. Staartjes VE, de Wispelaere MP, Vandertop WP, Schröder ML (2019) Deep learning-based preoperative predictive analytics for patient-reported outcomes following lumbar discectomy: feasibility of center-specific modeling. Spine J 19:853-861. https://doi. org/10.1016/j.spinee.2018.11.009

28. Hill AB (1965) THE ENVIRONMENT AND DISEASE: ASSOCIATION OR CAUSATION? Proc R Soc Med 58:295-300

29. Nemoto Y, Matsuzaki H, Tokuhasi Y et al (2006) Histological changes in intervertebral discs after smoking cessation: experimental study using a rat passive smoking model. J Orthop Sci 11:191-197. https://doi.org/10.1007/s00776-005-0987-4

30. Nasto LA, Ngo K, Leme AS et al (2014) Investigating the role of DNA damage in tobacco smoking-induced spine degeneration. Spine J 14:416-423. https://doi.org/10.1016/j.spinee.2013.08.034

31. Iwahashi M, Matsuzaki H, Tokuhashi Y et al (2002) Mechanism of Intervertebral Disc Degeneration Caused by Nicotine in Rabbits to Explicate Intervertebral Disc Disorders Caused by Smoking. Spine 27:1396-1401

32. Nachemson A, Elfström G (1970) Intravital dynamic pressure measurements in lumbar discs. A study of common movements, maneuvers and exercises. Scand J Rehabil Med Suppl 1:1-40

33. Wilke H-J, Neef P, Caimi M et al (1999) New In Vivo Measurements of Pressures in the Intervertebral Disc in Daily Life. Spine 24:755-762

34. Sørensen LT (2012) Wound Healing and Infection in Surgery: The Clinical Impact of Smoking and Smoking Cessation: A Systematic Review and Meta-analysis. Arch Surg 147:373-383. https://doi. org/10.1001/archsurg.2012.5

35. Shiri R, Lallukka T, Karppinen J, Viikari-Juntura E (2014) Obesity as a Risk Factor for Sciatica: A Meta-Analysis. Am J Epidemiol 179:929-937. https://doi.org/10.1093/aje/kwu007

36. Liuke M, Solovieva S, Lamminen A et al (2005) Disc degeneration of the lumbar spine in relation to overweight. Int J Obes 29:903-908. https://doi.org/10.1038/sj.ijo.0802974

37. Pinto ALDS, Holanda PMDB, Radu AS et al (2006) Musculoskeletal findings in obese children. J Paediatr Child Health 42:341344. https://doi.org/10.1111/j.1440-1754.2006.00869.x

38. Anandacoomarasamy A, Caterson I, Sambrook P et al (2008) The impact of obesity on the musculoskeletal system. Int J Obes 32:211-222. https://doi.org/10.1038/sj.ijo.0803715

39. van der Kraan PM, van den Berg WB (2007) Osteophytes: relevance and biology. Osteoarthr Cartil 15:237-244. https://doi.org/ 10.1016/j.joca.2006.11.006

40. Ghezelbash F, Shirazi-Adl A, Plamondon A et al (2017) Obesity and Obesity Shape Markedly Influence Spine Biomechanics: A Subject-Specific Risk Assessment Model. Ann Biomed Eng 45:2373-2382. https://doi.org/10.1007/s10439-017-1868-7

41. Gilleard W, Smith T (2007) Effect of obesity on posture and hip joint moments during a standing task, and trunk forward flexion motion. Int J Obes 31:267-271. https://doi.org/10.1038/sj.ijo. 0803430

42. Sharma A (2018) The Role of Adipokines in Intervertebral Disc Degeneration. Med Sci (Basel). https://doi.org/10.3390/medsc i6020034

43. Segar AH, Fairbank JCT, Urban J (2019) Leptin and the intervertebral disc: a biochemical link exists between obesity, intervertebral disc degeneration and low back pain - an in vitro study in a bovine model. Eur Spine J 28:214-223. https://doi.org/10.1007/ s00586-018-5778-7 
44. Müller-Lutz A, Schleich C, Schmitt B et al (2016) Gender, BMI and $\mathrm{T} 2$ dependencies of glycosaminoglycan chemical exchange saturation transfer in intervertebral discs. Magn Reson Imaging 34:271-275. https://doi.org/10.1016/j.mri.2015.10.024

45. Haneder S, Apprich SR, Schmitt B et al (2013) Assessment of glycosaminoglycan content in intervertebral discs using chemical exchange saturation transfer at 3.0 Tesla: preliminary results in patients with low-back pain. Eur Radiol 23:861-868. https://doi. org/10.1007/s00330-012-2660-6

46. Jiang J, Teng Y, Fan Z et al (2014) Does Obesity Affect the Surgical Outcome and Complication Rates of Spinal Surgery? A Metaanalysis. Clin Orthop Relat Res 472:968-975. https://doi.org/10. 1007/s11999-013-3346-3

47. Rihn JA, Kurd M, Hilibrand AS et al (2013) The Influence of Obesity on the Outcome of Treatment of Lumbar Disc Herniation.
J Bone Joint Surg Am 95:1-8. https://doi.org/10.2106/JBJS.K. 01558

48. Arts MP, Brand R, Akker VD et al (2011) Tubular Diskectomy vs Conventional Microdiskectomy for the Treatment of Lumbar Disk Herniation: 2-Year Results of a Double-Blind Randomized Controlled Trial. Neurosurgery 69:135-144. https://doi.org/10. 1227/NEU.0b013e318214a98c

Publisher's Note Springer Nature remains neutral with regard to jurisdictional claims in published maps and institutional affiliations. 